\title{
Mott-Hubbard transition in infinite dimensions
}

\author{
Ning-Hua Tong, ${ }^{1, *}$ Shun-Qing Shen, ${ }^{1,2, \dagger}$ and Fu-Cho $\mathrm{Pu}^{1,3}$ \\ ${ }^{1}$ State Key Laboratory of Magnetism, Institute of Physics, Chinese Academy of Sciences, P.O. Box 603-12, Beijing 100080, China \\ ${ }^{2}$ Department of Physics, The University of Hong Kong, Pokfulam Road, Hong Kong, China \\ ${ }^{3}$ Department of Physics, Guangzhou Normal College, Guangzhou 510400, China
}

(Received 17 May 2001; revised manuscript received 2 July 2001; published 27 November 2001)

\begin{abstract}
We analyze the unanalytical structure of metal-insulator transition (MIT) in infinite dimensions. A multiplevalued structure in Green's function and other thermodynamical quantities with respect to the interaction strength $U$ are observed at low temperatures by introducing a transformation into the dynamical mean-field equation of Hubbard model. A complete description of stable, metastable, and unstable phases is established in the regime $U_{c 1}(T)<U<U_{c 2}(T)$. The Maxwell construction is performed to evaluate the MIT line $U^{*}(T)$. We show how the first-order MIT at $U^{*}(T)$ for $T>0$ evolves into the second-order one at $U_{c 2}(0)$ for $T=0$. The phase diagram near MIT is presented.
\end{abstract}

DOI: 10.1103/PhysRevB.64.235109

PACS number(s): 71.30.+h, 71.27. $+\mathrm{a}, 71.28 .+\mathrm{d}$

\section{INTRODUCTION}

The Mott-Hubbard metal-insulator transition (MIT) is one of the classic topics in strongly correlated electron systems. ${ }^{1-3}$ The one-band Hubbard model is thought to be the minimum one such that the main features of the MIT are grasped. Early studies by Hubbard, and Brinkman and Rice explain very well the high and low energy behaviors of the local single-particle spectrum, respectively. ${ }^{4}$ In recent years, the transition between a Fermi-liquid phase and an insulating phase is extensively studied in the framework of dynamical mean-field theory (DMFT), ${ }^{5,6}$ by utilizing many methods such as iterative perturbation theory (IPT), ${ }^{7}$ quantum Monte Carlo (QMC) calculation, ${ }^{8-10}$ exact diagonalization (ED), ${ }^{11,12}$ projective self-consistent technique (PSCT),${ }^{13}$ and numerical renormalization group method. ${ }^{14,15}$ Below a critical temperature $T_{c},{ }^{8}$ a paramagnetic (PM) metallic phase and a PM insulating phase ${ }^{16}$ coexist in the regime $U_{c 1}(T)$ $<U<U_{c 2}(T),{ }^{10,15,17}$ where $U$ is the on-site Coulomb repulsion, and $U_{c 1}(T)$ and $U_{c 2}(T)$ are boundaries of the coexistence regime at temperature $T$. When $U$ increases, the metallic phase characterized by a finite density of spectrum at the Fermi level disappears discontinuously at $U_{c 2}(T)$. On the other hand, the insulating phase in the large $U$ regime is destroyed abruptly when $U$ decreases to $U_{c 1}(T) .{ }^{15}$ Between $U_{c 1}(T)$ and $U_{c 2}(T)$ lies a first-order MIT line $U^{*}(T)$, at which the free energies of the two coexisting phases are equal. ${ }^{10,17}$ At the critical temperature $T_{c}$, both $U_{c 1}(T)$ and $U_{c 2}(T)$ equal to $U_{c}$ and a second-order transition occurs. Above $T_{c}$, when $U$ increases, the system changes from metallic into insulating phase through crossover. ${ }^{17,18}$ At zero temperature, it is found that the metallic phase has a lower energy in the coexisting regime. The MIT occurs at $U$ $=U_{c 2}(0)$ in such a way that the energy difference between the two coexisting solutions disappears quadratically, i.e., $E_{g}^{I}-E_{g}^{M} \sim\left[U_{c 2}(0)-U\right]^{2} .^{13}$

Despite the enormous efforts on this problem, there is no complete description for the stable, metastable, and unstable phases. These phases always appear near the first-order phase transition and are important for understanding the be- haviors of the system under external influences. Traditionally, the first-order nature of MIT is disclosed from the hysteresis or discontinuity in some relevant physical quantities with respect to $U$. In this way, the unanalytical feature of these quantities is not displayed, and the Maxwell construction cannot be performed explicitly to produce the MIT line $U^{*}(T)$. Hence the MIT deserves further study in this direction. In this paper, a transformation is introduced to the DMFT self-consistent equation to investigate the unanalytical structure in the Green's function, local density of states (DOS) and double occupation probability in terms of $U$. For each $U$ in the regime $U_{c 1}(T)<U<U_{c 2}(T)$, three solutions arise: stable, metastable, and unstable non-Fermi-liquid metallic solutions. The Maxwell construction is performed to produce the MIT line $U^{*}(T)$. The second-order MIT at $T$ $=T_{c}$ is found to persist at a metastable level in the low temperature limit. When temperature approaches zero, the first-order transition at $U^{*}(T)$ evolves into second-order one $^{19}$ at $U_{c 2}(0)$. Finally an ED phase diagram of MIT is presented.

\section{MODEL AND METHOD}

We start with the single-band Hubbard model at halffilling for the PM solution:

$$
H=-t \sum_{\langle i, j\rangle \sigma} c_{i \sigma}^{\dagger} c_{j \sigma}+\sum_{i} U n_{i \uparrow} n_{i \downarrow} .
$$

The notations are conventional. A semicircular bare density of states is used: $D(\varepsilon)=\left(2 / \pi W^{2}\right) \sqrt{W^{2}-\varepsilon^{2}}$, and $W=1.0$ is taken as the energy unit. To introduce our method, denote a thermodynamical quantity by $Q$. For a specific temperature, the formal dependence of $Q$ on the on-site interaction $U$ is written as $Q=f_{Q}(U)$. We consider the analogy between MIT and conventional liquid-gas transition, which was put forward by Castellani et al. ${ }^{20}$ In this analogy, $U$ corresponds to the pressure $P$ as the driving force of phase transition, and $D=\left\langle n_{\uparrow} n_{\downarrow}\right\rangle$ corresponds to the inverse density $v$. This analogy is helpful for us to understand the characters of MIT. ${ }^{8}$ In the mean-field treatment of MIT, unanalyticity of thermody- 
namical quantities versus $U$ should appear in a similar way as that appears in the isotherm $v=v(P)$ of van der Waals equation. In this way, we realize that for an appropriate quantity $Q$, the function $f_{Q}(U)$ should be continuous, but may have " $Z$ "- or " $S$ "-shaped structure. Now we focus on the Green's function at an imaginary time $\tau$. As an example, we choose $Q=G(\beta / 2)$. A discontinuous jump in $G(\beta / 2)$ was observed when we directly swept along $U$ axis. In order to avoid such discontinuity, instead of directly calculating $G(\beta / 2)=f_{G(\beta / 2)}(U)$ for each $U$, we try to find a solution of $G(\beta / 2)$ for the transformed self-consistent equation,

$$
G(\beta / 2)=f_{G(\beta / 2)}\{U-\lambda[A-G(\beta / 2)]\},
$$

where the parameters $A$ and $\lambda$ are assigned such that $G(\beta / 2)$ is single valued with respect to $U$ even if the function $f_{G(\beta / 2)}(U)$ has a " $Z$ "- or " $S$ "-shaped structure. Existence of such an unanalytical structure is strongly indicated by the discontinuity of $G(\beta / 2)$. After the new equation is solved, the original functional dependence $G(\beta / 2)=f_{G(\beta / 2)}\left(U^{\prime}\right)$ is recovered by plotting the self-consistent solution $G(\beta / 2)$ versus the argument $U^{\prime}=U-\lambda[A-G(\beta / 2)]$. In practice, Eq. (2) is combined with the DMFT self-consistent equations by iterative calculation. For fixed $U, A$, and $\lambda$, in each iteration, we first calculate $U^{\prime}=U-\lambda[A-G(\beta / 2)]$, where $G(\beta / 2)$ is taken from previous iteration. Take the chemical potential $\mu=U^{\prime} / 2$ to realize the constraint of half-filling. Then $U^{\prime}$ is used to replace $U$ in the conventional algorithm ${ }^{6}$ to proceed with the calculation in this iteration (or from initialization). At the end, besides the new set of parameters for the effective single-impurity Anderson model, a new $G(\beta / 2)$ is also produced. Both of them are used in next iteration. $U^{\prime}$ varies with iteration until the Green's function converges. Then both Eq. (2) and the DMFT equations will be fulfilled. No additional computational effort is needed in implementing our scheme. After the convergence is reached, other quantities, such as $G(\tau)$ for a general $\tau$, the double occupancy $\left\langle n_{\uparrow} n_{\downarrow}\right\rangle$, and the local DOS at the Fermi surface $\rho(0)$, can be calculated from the converged Green's function $G\left(i \omega_{n}\right)$ and $U^{\prime}$. The whole functional dependence $Q$ $=f_{Q}\left(U^{\prime}\right)$ can be obtained by sweeping $U$ in Eq. (2). This modified self-consistency scheme was used in the study of phase separation in double-exchange systems. ${ }^{21}$ This method is also effective to reveal the unanalyticity in MIT, and the final results are essentially independent of the selection of $A$ and $\lambda$ if only $|\lambda|$ is large enough to "stretch" the curve. In this paper, the finite-temperature ED technique is used to implement the above scheme. ${ }^{22}$ This technique, originally proposed by Caffarel and Krauth, ${ }^{11}$ uses $\chi^{2}$ fit to obtain new parameters of the effective Anderson impurity model in the iteration process.

\section{RESULTS AND DISCUSSION}

The minus imaginary-time Green's function at $\tau=\beta / 2$ is plotted versus $U$ in Fig. 1(a) for $T=0.01$, which is lower than the critical temperature $T_{c}$. It is shown that $-G(\beta / 2)=$ $-f_{G(\beta / 2)}(U)$ is indeed continuous with a " $Z$ "-shaped structure. Three solutions of $G(\beta / 2)$ coexist in an extent of $U$. At
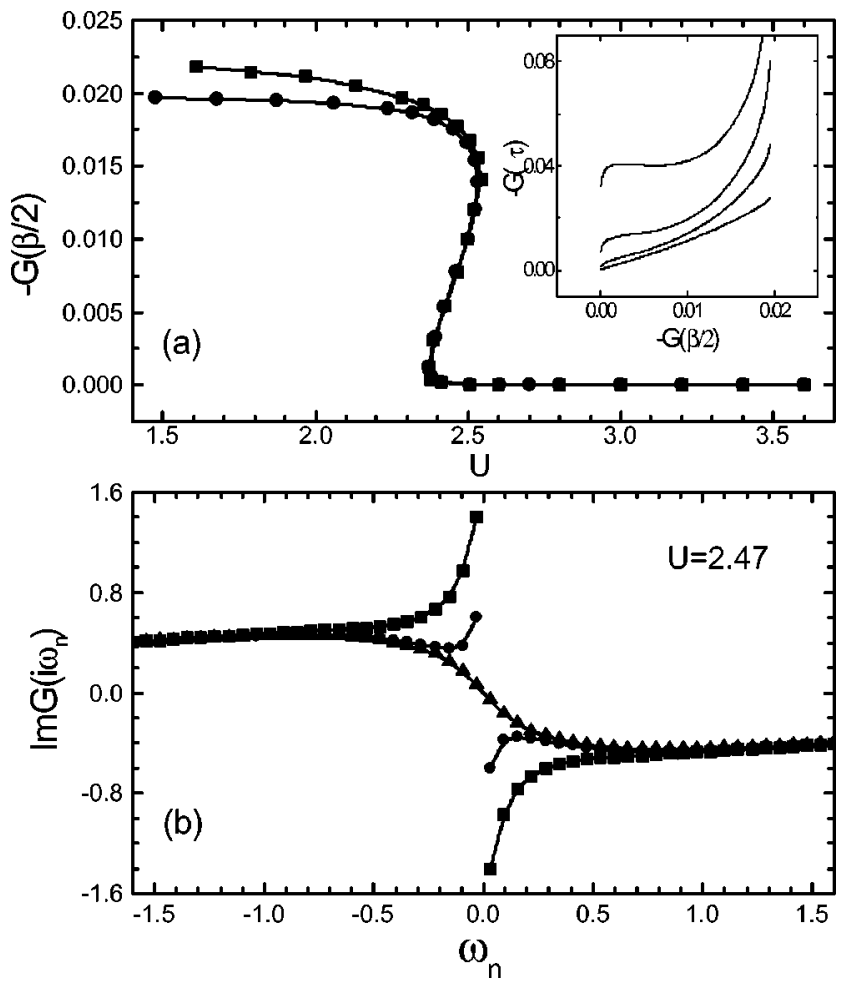

FIG. 1. (a) $U$-dependence of minus imaginary-time Green's function $-G(\tau)$ at $\tau=\beta / 2$, obtained using $N s=5$ (square) and $N s=6$ (dot). (b) The three coexisting solutions of Green's function on Matsubara frequency axis, obtained using $N s=6$ at $U=2.47$. Both figures are for $T=0.01$, and the thin lines are for guiding eyes. Inset of (a) $-G(\tau)$ versus $-G(\beta / 2)$ as $U$ varies $(N s=6)$. From top to bottom, $\tau=\beta / 32, \beta / 16, \beta / 8, \beta / 4$.

$T=0.01$, the results evaluated by ED technique of five and six sites are very well consistent. We find that our results are independent of sufficiently large $\lambda$, the value of $A$, or the initial seeding of iteration. We plot $G(\tau)$ versus $G(\beta / 2)$ as shown in the inset of Fig. 1(a). The analytical behaviors of these curves appear clearly that for each $\tau \in[0, \beta], G(\tau)$ is unanalytical in terms of $U$. Moreover, the coexistence boundaries of $G(\tau)$ have the same values irrespective of $\tau$. The Fourier transformation $G\left(i \omega_{n}\right)$ as well as all other quantities will have the same boundary of coexistence, if only they depend on the Green's functions analytically. So, the boundaries of coexistence $U_{c 1}(T)$ and $U_{c 2}(T)$ are well defined in MIT.

In Fig. 1(b), three coexisting solutions of the Matsubara Green's function at $T=0.01$ and $U=2.47$ are presented. The upper and the lower curves are similar with those from QMC. ${ }^{8,10}$ One is metallic-like (squares) and the other is insulating-like (up triangles). A new metallic-like solution (dots) is found between them. At finite temperatures, this newly discovered solution has the highest free energy among the threes and is unstable. In this paper, we do not intend to quest for the highest numerical precision, but focus on the qualitative features of the Mott-Hubbard transition and its temperature evolution. All our results in the following are obtained using $N s=6$ ( $N s$ is the number of sites).

The minus imaginary-time Green's function $-G(\tau)$ at $\tau$ 


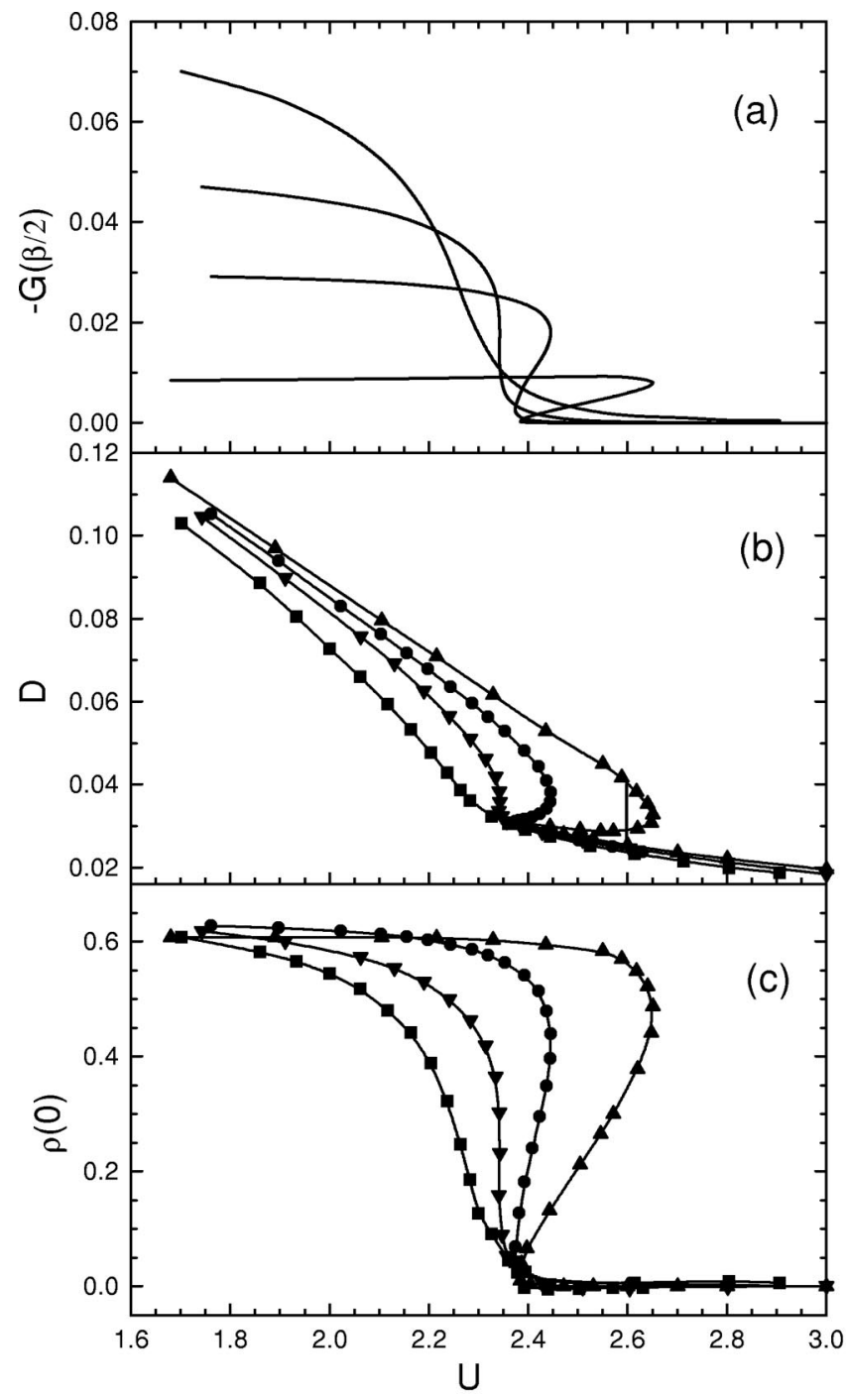

FIG. 2. (a) $U$-dependence of $-G(\beta / 2)$ at $T=0.04,0.025$, 0.015 , and 0.005 . (From top to bottom on the left-hand side.) (b) and (c) Double occupancy $D$ [in (b)] and DOS at Fermi surface $\rho(0)$ [in (c)] versus $U$ at $T=0.04$ (squares), 0.025 (down triangles), 0.015 (dots), and 0.005 (up triangles). Thin lines are for guiding eyes. The thick vertical line in (b) shows Maxwell construction for $T=0.005$. In small $U$ regime, the minor deviation of $\rho(0)$ at $T$ $=0.005$ from $2 / \pi W$ originates from the finite-size effect.

$=\beta / 2$, the double occupancy $D=\left\langle n_{\uparrow} n_{\downarrow}\right\rangle$ and the local DOS at Fermi surface $\rho(0)$ are plotted versus $U$ for several temperatures in Figs. 2(a), 2(b), and 2(c), respectively. In Fig. $2(\mathrm{a}),-G(\beta / 2)$ decreases monotonously when $U$ increases at $T=0.04$. As the temperature decreases, it decays more rapidly in the intermediate $U$ regime. At $T=0.025$, a singular point arises at $U=2.34$ where the slope diverges. Below this temperature, the curve is still continuous, but has a " $Z$ "-shaped structure. With decreasing temperature, the curve is compressed along $-G(\beta / 2)$ axis and the coexisting regime extends along the $U$ axis. This leads to a more pronounced multiple-valued structure. We do not find slowing down of this tendency as temperature is lowered down to $T$ $=0.0025$. Similar temperature evolution behaviors are also observed in $D-U$ [Fig. 2(b)] and $\rho(0)-U$ [Fig. 2(c)] curves. Within our numerical precision, the three kinds of curves produce the same value of critical point $\left(U_{c}, T_{c}\right)$ and the same boundaries of coexistence regimes for $T<T_{c}$. This is consistent with our conclusion that the unanalytical structure is universal for all thermodynamical quantities. The critical temperature $T_{c}$ and interaction $U_{c}$ are thus estimated to be $T_{c} \approx 0.025, U_{c} \approx 2.34$, which agree quite well with that obtained from QMC: $T_{c}=0.026 \pm 0.003, U_{c}=2.38 \pm 0.02 .{ }^{8}$ At the critical point, all three quantities have divergent slopes with respective to $U$. From Figs. 2(a)-2(c), the boundaries of coexistence regime $U_{c 1}(T)$ and $U_{c 2}(T)$ are easily obtained, in contrast to previous approaches. ${ }^{10,15,17}$

The double occupancy $D$ is of special thermodynamical significance. The free energy can be evaluated by integrating along the $D-U$ lines in Fig. 2(b):

$$
F(U, T)=F(0, T)+\int_{0}^{U} D\left(U^{\prime}, T\right) d U^{\prime} .
$$

In Fig. 2(b), $D-U$ curves have similar unanalytical behavior with $v-P$ isotherms of van der Waals equation. At $T \geqslant T_{c}$, the curves of $D-U$ are similar with those from $\operatorname{IPT}^{23}$ and QMC. ${ }^{8}$ The Mott critical point $\left(U_{c}, T_{c}\right)$ was studied in detail by $\mathrm{QMC}^{8}$ and Landau theory of phase transition. ${ }^{23}$ At 0 $<T<T c$, three solutions coexist in a regime around $U_{c}$. We compare free energies of the three solutions for a fixed $U$ and find that the phase with intermediate $D$ has the highest free energy. For the metallic (with largest $D$ ) and the insulating (with smallest $D$ ) phases, their free energies cross at the point $U=U^{*}(T)$, i.e., $F_{M}\left(U^{*}, T\right)=F_{I}\left(U^{*}, T\right)$. We solved this equation numerically to determine $U^{*}(T)$. As $U$ passes by $U^{*}$ from below, a stable metallic phase $\left[F_{M}(U, T)\right.$ $\left.<F_{I}(U, T)\right]$ transits into a stable insulating phase $\left[F_{I}(U, T)<F_{M}(U, T)\right]$ (see Fig. 3). This transition is accompanied with a finite jump $\Delta D$ of the double occupancy $D$, which is determined by the Maxwell construction line as shown in Fig. 2(b). Hence the MIT at finite temperatures is identified as a generic first-order phase transition. At $T$ $<T_{c}$, it is interesting that there is a discontinuous jump in the slope of the $D-U$ curve at $U=U_{c 1}(T)$. At this point, $\partial F / \partial U=D$ is continuous but $\partial^{2} F / \partial U^{2}=\partial D / \partial U$ is discontinuous. It means that the transition is of second order. This singularity is directly evolved from that of the critical point $\left(U_{c}, T_{c}\right)$ as temperature decreases. It turns out that the second-order MIT at $T=T_{c}$ does not disappear in the regime $T<T_{c}$, but persists to the absolute zero temperature at a metastable level. This feature of MIT is not unique among the first-order phase transitions. In the double-exchange model for manganites, a similar feature was observed in the isotherm of charge density $n$ versus chemical potential $\mu$ (see Fig. 2 in Ref. 21). There, when the phase separation (also a typical first-order phase transition) between paramagnetic (PM) and ferromagnetic (FM) phases appears at $T$ $<T_{c}$, the second-order PM-FM transition persists at the metastable level down to zero temperature.

Now we discuss the phenomenon of critical slowing down. In a previous QMC study of MIT, ${ }^{10}$ the critical slowing down in the convergence of iteration was observed near the boundaries of coexistence regime, and it was used as the 


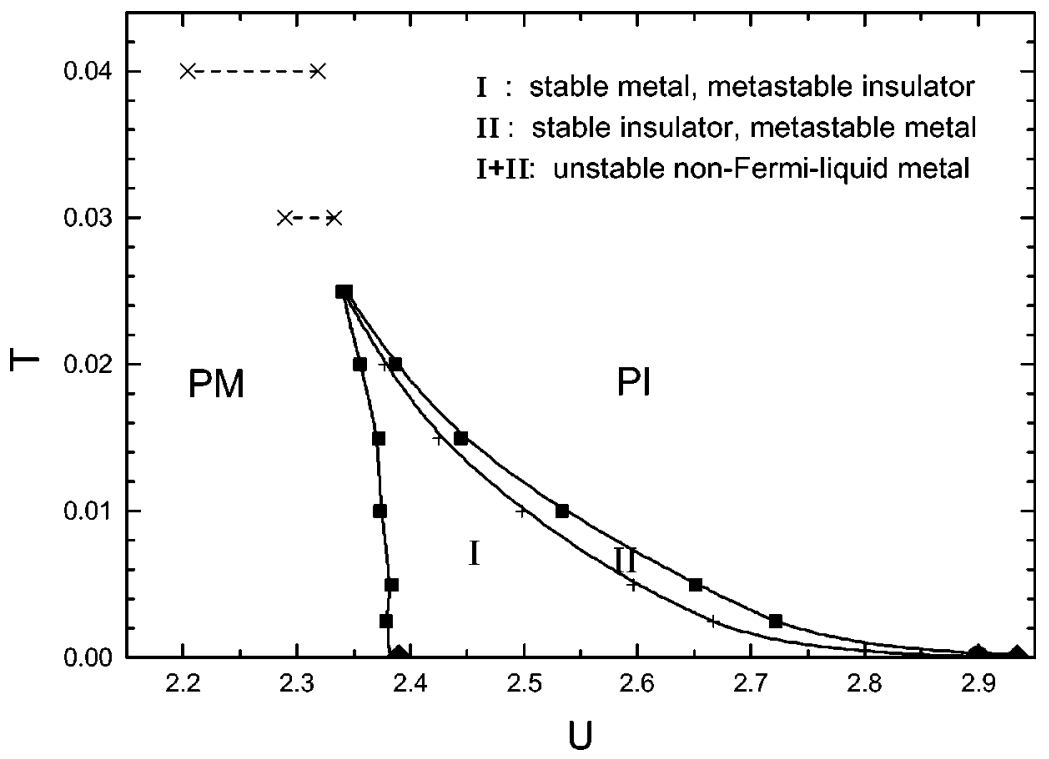

FIG. 3. Phase diagram of Mott-Hubbard transition obtained from ED method. The squares and crosses mark out the coexistence boundaries and the MIT line $U^{*}(T)$, respectively. Thin lines are eye-guiding lines. Crosses with horizontal dashed lines above $T_{c}$ show the crossover regime. PSCT (Ref. 13) (circle) and NRG (Ref. 14) (diamonds) results for $T=0$ are also shown. PM, PI denote paramagnetic metal and paramagnetic insulator, respectively. The properties of phases in area I, II and $\mathbf{I}+\mathbf{I I}$ are noted in the figure. indicator to determine these boundaries. Such critical slowing down arises as one tries to directly calculate the " $\mathrm{Z}$ "' shaped curve for the first-order phase transition. In our calculations we did not observe the critical slowing down of the same kind since what we practically calculated is a "stretched" single-valued function $f_{Q}[U-\lambda(A-G(\beta / 2)]$, and the coexistence of solutions is removed by the transformation. In contrast, we observed a weak slowing down of convergence at the singular point at $U=U_{c 1}(T)$ where the slope of $D-U$ curve is discontinuous. This slowing down is associated with the metastable second-order phase transition identified above. Hence it is different from that discussed in Ref. 10.

At zero temperature, Rozenberg ${ }^{12}$ and Moeller $^{13}$ found a second-order MIT at $U_{c}=U_{c 2}(0)$. Here, we discuss how the first-order MIT at finite temperature evolves into the secondorder one at zero temperature. Figure 2(b) shows that when temperature decreases, the linear behavior of $D-U$ curve becomes dominant and the intermediate branch of $D$ goes closer to the insulating solution. As a result, the first-order transition point $U^{*}(T)$ moves towards $U_{c 2}(T)$ according to the Maxwell construction. This tendency continues when the lowest temperature $T=0.0025$ in this paper is reached. It is anticipated that at $T=0$, the intermediate branch of $D$ merges with the lowest branch and they become degenerate. The linear metallic $D-U$ line meets the insulating branch at $U_{c 2}(0)=U^{*}(0)$, where the jump of $D$ disappears. Hence the transition is of second order at $T=0$, and it is consistent with the $D-U$ curves shown in Fig. 34 of Ref. 6. The quadratic relation $E_{g}^{I}-E_{g}^{M} \sim\left[U_{c 2}(0)-U\right]^{2}$ (Ref. 12) is obtained by assuming a strict linear $D-U$ curve at $T=0$. Here it is worth pointing out that the term second-order transition in this context means that at $U^{*}(0), \partial E / \partial U$ is continuous and $\partial E^{2} / \partial^{2} U$ is discontinuous, where $E=\min \left(E_{g}^{I}, E_{g}^{M}\right)$. This transition is special in that metastable state still exists near the transition point at zero temperature.

In Fig. 2(c), the local DOS at the Fermi surface $\rho(0)$ is calculated by extrapolating $-\operatorname{Im} G\left(i \omega_{n}\right) / \pi$ to the limit $\omega_{n}$ $\rightarrow 0^{+}$. In the small $U$ regime, $\rho(0)$ does not change with interaction and remains its noninteracting value $2 /(\pi W)$, as required by Luttinger theorem for momentum independent self-energy. ${ }^{5}$ At temperatures above $T_{c}$ [e.g., the curve for $T=0.04$ in Fig. 2(c)], the Fermi-liquid phase in small $U$ regime evolves continuously into an insulating phase in large $U$ regime through a crossover. At temperatures below $T_{c}$, the behavior of $\rho(0)$ in the small and large $U$ regime are similar with previous results. ${ }^{6,15}$ In the coexisting regime, the metallic phase at $U_{c 2}$ is smoothly connected to the insulating phase at $U_{c 1}$ through a non-Fermi-liquid phase. According to the integral along the $D-U$ lines, this non-Fermi-liquid phase has the highest free energy. In light of the present result, the discontinuity and hysteresis of $\rho(0)$ in Ref. 15 originates from numerical instabilities at the boundaries of the coexisting regime. Physically, such instabilities reflect the unstable character of the metastable phases near phase boundaries. The metastable second-order transition at $U_{c 1}(T)$ is now identified as the transition between metallic $(\rho(0)>0)$ and insulating $[\rho(0) \approx 0]$ phases. When temperature decreases, the intermediate solution of $\rho(0)$ moves downwards, but there is no obvious tendency that it will merge with the insulating solution of $\rho(0)$ in the limit $T$ $\rightarrow 0$. This is in contrast to the $D-U$ curve as shown in Fig. 2(b). From Fig. 2(c), we conclude that in the low temperature limit, as $U$ passes by $U_{c 2}(0)$ from below, $D$ varies continuously but $\rho(0)$ will jump to zero from a finite value as was observed in NRG study. ${ }^{14}$ Other quantities characterizing the MIT such as the quasi-particle weight $Z$ (Ref. 11) and local spin-spin correlation $\left\langle M_{z}(\tau) M_{z}(0)\right\rangle$ (Ref. 17) should have similar unanalyticity in terms of $U$.

Based on the analysis of the Green's function and other physical quantities, we can determine the phase diagram near the MIT. In Fig. 3, two boundaries of the coexistence regime meet at a critical point $U_{c} \approx 2.34, T_{c} \approx 0.025$. Above this point, the metallic phase in small $U$ regime evolves into an insulating phase in large $U$ regime through a crossover area. Following Ref. 15, the $U$ regime where $-d \rho(0) / d U$ is larger than its half maximum is regarded as the crossover regime. Below $T_{c}$, between the two boundary lines three 
phases coexist, i.e., stable, metastable, and unstable phases. Positions and properties of these phases are labeled in Fig. 3. The MIT line $U^{*}(T)$ from Maxwell construction resembles that obtained by Joo et al. ${ }^{10}$ Compared with the phase diagram of other authors, the $U_{c 2}(T)$ line agrees pretty well with NRG results, ${ }^{15}$ and the $U_{c 1}(T)$ line lies between that from $\mathrm{NRG}^{15}$ and $\mathrm{QMC}^{10}$ studies. Although the lowest temperature in the present paper is $T=0.0025$, our phase boundaries extrapolated to zero temperature is in good consistency with the results of NRG (Ref. 14) and PSCT. ${ }^{13}$

\section{SUMMARY}

In summary, we introduce a transformation into the DMFT self-consistent equations to study the unanalytical behavior of thermodynamical quantities in the Mott-Hubbard metal-insulator transition in infinite dimensions. Using the

*Electronic mail: tongnh@aphy.iphy.ac.cn

†Electronic mail: sshen@hkucc.hku.hk

${ }^{1}$ N. F. Mott, Proc. Phys. Soc. London, Sect. B 62, 416 (1949); Metal-Insulator Transitions, 2nd ed. (Taylor \& Francis, London, 1990).

${ }^{2}$ F. Gebhard, The Mott Metal-Insulator Transition, Springer Tracts in Modern Physics Vol. 137 (Springer, Berlin, 1997).

${ }^{3}$ D. B. McWhan and J. P. Remeika, Phys. Rev. B 2, 3734 (1970); D. B. McWhan et al., ibid. 7, 1920 (1973).

${ }^{4}$ J. Hubbard, Proc. R. Soc. London, Ser. A 281, 401 (1964); W. F. Brinkman and T. M. Rice, Phys. Rev. B 2, 4302 (1970).

${ }^{5}$ W. Metzner and D. Vollhardt, Phys. Rev. Lett. 62, 324 (1989).

${ }^{6}$ A. Georges, G. Kotliar, W. Krauth, and M. J. Rozenberg, Rev. Mod. Phys. 68, 13 (1996).

${ }^{7}$ A. Georges and G. Kotliar, Phys. Rev. B 45, 6479 (1992).

${ }^{8}$ M. J. Rozenberg, R. Chitra, and G. Kotliar, Phys. Rev. Lett. 83, 3498 (1999).

${ }^{9}$ W. Krauth, Phys. Rev. B 62, 6860 (2000).

${ }^{10}$ J. Joo and V. Oudovenko, cond-mat/0009367.

${ }^{11}$ M. Caffarel and W. Krauth, Phys. Rev. Lett. 72, 1545 (1994).

${ }^{12}$ M. J. Rozenberg, G. Moeller, and G. Kotliar, Mod. Phys. Lett. B 8, 535 (1994).

${ }^{13}$ G. Moeller, Q. Si, G. Kotliar, M. Rozenberg, and D. S. Fisher,
ED technique at finite temperatures, the " $Z$ "'-shaped multiple-valued structure of several quantities in terms of $U$ is obtained. An unstable non-Fermi-liquid phase as well as the two phases discovered previously are found in the regime $U_{c 1}(T)<U<U_{c 2}(T)$. The MIT line $U^{*}(T)$ is obtained by Maxwell construction. The second-order MIT at $T=T_{c}$ persists at the metastable level down to zero temperature, and the first-order MIT at $U^{*}(T)$ evolves into second-order one at $U_{c 2}(0)$ when temperature approaches zero. The method used in this paper should be useful for the study of other first-order phase transitions.

\section{ACKNOWLEDGMENT}

This work was supported by a CRCG of the University of Hong Kong and a RGC grant of Hong Kong.

Phys. Rev. Lett. 74, 2082 (1995).

${ }^{14}$ R. Bulla, Phys. Rev. Lett. 83, 136 (1999).

${ }^{15}$ R. Bulla, T. A. Costi, and D. Vollhardt, Phys. Rev. B 64, 045103 (2001).

${ }^{16}$ Although an insulator is only strictly defined for $T=0$, we use the term insulating or insulating-like to denote the solution with vanishingly small spectral weight at the Fermi level for $T>0$.

${ }^{17}$ M. J. Rozenberg, G. Kotliar, and X. Y. Zhang, Phys. Rev. B 49, 10181 (1994).

${ }^{18}$ M. J. Rozenberg, G. Kotliar, H. Kajueter, G. A. Thomas, D. H. Rapkine, J. M. Honig, and P. Metcalf, Phys. Rev. Lett. 75, 105 (1995).

${ }^{19}$ As to be discussed in the following, the MIT at $T=0$ is of second order only in the sense that at the transition point, $\partial E / \partial U$ is continuous and $\partial E^{2} / \partial^{2} U$ is not. It is special and should be distinct from the conventional second-order transition.

${ }^{20}$ C. Castellani, C. Di Castro, D. Feinberg, and J. Ranninger, Phys. Rev. Lett. 43, 1957 (1979).

${ }^{21}$ N. H. Tong and F. C. Pu, Phys. Rev. B 62, 9425 (2000).

${ }^{22}$ Part of the programs provided by Ref. 6 were adapted for our use.

${ }^{23}$ G. Kotliar, E. Lange, and M. J. Rozenberg, Phys. Rev. Lett. 84, 5180 (2000). 